\title{
AUTORINNEN UND AUTOREN \\ LES AUTEURS
}

Olivia CARPI, maître de conférences à l'université de Picardie - Jules Verne, Amiens

Vincent CHALLET, maître de conférences à l'université Paul-Valéry - Montpellier III, UFR des sciences humaines et sciences de l'environnement, département d'histoire, Montpellier

Jean-Claude CheYNeT, professeur d'histoire médiévale, université de ParisSorbonne - Paris IV

Philippe DEPREUX, professeur d'histoire médiévale, membre de l'IUF, université de Limoges, faculté des lettres et des sciences humaines, Limoges

Kai-Henrik GÜNTHER, wissenschaftlicher Mitarbeiter an der Universität Konstanz, Lehrstubl für die Geschichte der Religionen und des Religiösen in Europa

Uwe ISRAEL, Direktor des Deutschen Studienzentrums in Venedig

François MARTIN, directeur d'études à l'École pratique des hautes études, section des sciences historiques et philologiques, Paris.

Steffen PATZOLD, Professurvertreter für mittelalterliche Geschichte, Historisches Seminar der Eberhard-Karls-Universität Tübingen

Jörg PELTZER, Gruppenleiter der Emmy-Noether Gruppe »Rang und Ordnung«, am Institut für fränkisch-pfälzische Geschichte und Landeskunde, Heidelberg

Bruno PoTTIER, maître de conférences à l'université de Provence - AixMarseille I, UFR civilisation et humanités, département d'histoire, Aix-enProvence

Marianne SAGHY, maître de conférences an der Central European University, Department of Medieval Studies, Budapest

Alain SAINT-DENIS, professeur d'histoire médiévale à l'université de Bourgogne, UFR des sciences humaines, Dijon

Werner TROSSBACH, Professor für Agrargeschichte an der Universität Kassel

Hanna VOLLRATH, em. Universitätsprofessorin für mittelalterliche Geschichte an der Ruhr-Universität Bochum

Claudia ZEY, Professorin für mittelalterliche Geschichte am Historischen Seminar der Universität Zürich 


